\title{
Implementing an Education Program in Elementary Schools to Improve Healthy Food Choices
}

\author{
Tonia Mailow*
}

Murray State University, Nursing 43 Northshore Circle Murray, KY-42071, United States

*Corresponding author: Tonia Mailow, Murray State University Nursing 43 Northshore Circle Murray, KY-42071, United States, Tel: 2709786991,2708096678; Fax: 2708096661; E-mail: tmailow@murraystate.edu

Received date: April 28, 2017; Accepted date: May 08, 2017; Published date: May 15, 2017

Citation: Tonia Mailow (2017) Implementing an Education Program in Elementary Schools to Improve Healthy Food Choices. J Child Obes 2: 9.

Copyright: (c) 2017 Mailow T. This is an open-access article distributed under the terms of the Creative Commons Attribution License, which permits unrestricted use, distribution, and reproduction in any medium, provided the original author and source are credited.

\section{Abstract}

Childhood obesity is a major concern in the United States. Research suggests that nutrition education is needed to improve the knowledge of children about healthy nutrition choices. The need for clarity of lifestyle behaviors and education of children is vital and must be addressed. The purpose of this project was to provide an educational program specific to healthy food choices to elementary school teachers for implementation in the classroom and to increase the knowledge of elementary school teachers about reading food label and selecting healthier choices. Results of this project support the need for education on healthy food choices and reading food labels. Pre-and-posttest $t$ tests were conducted on mean scores to measure nutritional knowledge. An increase in scores suggested that the use of nutrition education can be successful in improving the knowledge of teachers about how to make healthy food choices and read food labels.

Keywords: Childhood obesity; Lifestyle behaviors; Healthy food; Obese children

\section{Implementing an Education Program on Healthy Food Choices in Elementary Schools}

Childhood obesity is a health problem affecting $18 \%$ of US children ages $6-11$ years [1]. In the past 25 years, the prevalence of obesity in children has more than tripled as the prevalence of overweight young children has increased [2]. Obese children are at a greater risk for cardiovascular disease and studies have identified as many as $7 \%$ of obese children with one or more risk factors for cardiovascular disease [3]. Children who are obese are less active and unable to participate in activities like their peers. They tend to be socially awkward with other children leading to isolation from peers. Additionally, overweight and obese children tend to become adults who are overweight and obese [4]. Obesity is related to more general practitioner visits, more school absences, more health-related limitations and overall lower general health scores [4]. some of the more prevalent chronic diseases associated with obesity include: Increased blood pressure, increased cholesterol, heart disease and diabetes [5].

Obesity in the U.S. continues to increase and healthy people 2010 goals calling for a $6 \%$ decrease in obesity were unmet. Healthy People 2020 goals have increased the responsibility of health care providers and state agencies to assist in reducing the percentage of children, ages 6-11, diagnosed as obese [6]. The American Medical Association (AMA) now recognizes obesity as a disease and provides clear guidelines for identification and reimbursement for interventions. However, education to prevent obesity is lacking. The need for education addressing healthy food choices with school age children is vital as a beginning intervention to decrease overweight and obese children and reach 2020 health goals.

Children's food choices are influenced by many external factors. Food and beverage marketing target children under the age of 12 [7]. An average of about $\$ 10$ billion is spent to market products to children, with four of the top ten items being available without parent permission [7]. The information being received by this age group is through mass marketing of products often high in calories and deficient in nutritional value. Children need consistent nutritional education in an environment where it can be role modeled and reinforced.

The literature supports schools as vital to educating students on how to eat appropriately and choose the best meal [5] it is important that children are educated about healthy choices to reduce their risk of obesity-related diseases. The purpose of this project is to provide an educational program specific to healthy food choices to elementary school teachers for implementation in the classroom.

\section{Literature Review}

School nutrition programs are effective tools for preventing childhood obesity in the U.S. [8] Children need education about the importance of healthy food choices. Targeting diet needs and educating on healthy food choices can help in the struggle to improve the health of children. A 2-years based school-based intervention in Spain was implemented. Results showed a $62 \%$ decrease in the prevalence of overweight children [8]. 
Interventions to improve the education of children on healthy choices, especially food choices, is the first step in the process to decrease the number of obese and overweight children.

Ling et al. [8] investigated lifestyle interventions with the purpose of improving health behaviors among elementary school children in rural KY. A total of 1508 children ( 804 boys and 679 girls) in grades K-5 at 4 elementary schools participated. Nutrition knowledge was one of the four goals developed to improve health outcomes [9]. This project allowed educators to participate in the education of educators. An increase in knowledge and awareness of the importance of healthy food choices were the part of the goals.

Nutritional intake of healthy foods, such as fruits and vegetables was measured once a month for five months during the study ( $t 1, t 2, t 3$, and $t 4)$ [8]. The school-based healthy lifestyle intervention was developed based on the principles of the Coordinated School Health Program recommended by the Center for Disease Control. Nutrition was assessed using the School Physical Activity and Nutritional (SPAN) questionnaire. School personnel also participated in six Healthier U.S. School Challenge Nutrition curriculum workshops and attended 1 nutrition conference [9]. This would allow the increase of knowledge of the personnel involved in the long term improvement of nutritional education in the schools. The personnel were also provided with one day of intensive classroom nutrition education and used that information to help incorporate nutrition education into the school curriculum [9].

At baseline, 206 (14.8\%) of the participants met the recommended serving of 3 or more vegetables and 2 or more fruits per day. [9] Using a one-way ANOVA analysis, subsequent data found statistically significant increases in the number of participants that met the recommended daily requirements for vegetables and fruit: $\mathrm{t} 1=209(15.3 \%), \mathrm{t} 2=384$ (27.85), $\mathrm{t} 3=410$ $(30.2 \%)$ and $\mathrm{t} 4=347$ (25.9\%) respectively, $\mathrm{p}<0.001$ [8]

Prosper et al. [9] also explored the dietary habits of children. A total of 1469 students, in 51 elementary, middle and high schools participated. Schools that were considered Title I Status (40\% of enrollment consists of children from low income families) were enrolled in the study. The intervention, titled Healthy for Life/PE4ME focused on nutrition and two other areas, environment and physical activity [10]. The program used the School Health Index (SHI) to assist in the guidance of the evaluation and improvement of healthy nutrition. Nutrition education was integrated into the school curriculum and taught over a one year period by the PE teacher. A registered dietician incorporated a two hour-long nutrition project in after school sessions, during the same time period. Course content stressed the importance of eating a healthy breakfast, increasing the intake of fruits and vegetables and different strategies to help prepare healthy meals, decreasing the amount of fat and sugar, how to eat out healthy and how to read a nutrition label [10].

At the beginning of the school year, PE teachers completed six hours of training to assist in the facilitation of the curriculum. Data were collected three times throughout the project, with the assessment occurring at the beginning of the program, midyear and year end. Questionnaires were utilized to collect data.
The first was an eight-item lifestyle questionnaire. It included questions pertaining to dietary habits (frequency of vegetable, fruit, breakfast, milk consumption and the consumption of fast food). Another questionnaire targeted specifically at preschool and elementary school students measured knowledge of healthy food behaviors in children. Twelve photo pairs were used and included one healthy food and one unhealthy food. Students were asked to choose the healthy food. Correct answers were totaled and a score, ranging from 0-12 was given to each student. At baseline, data collection was specific to students making healthy food choices. Subsequent data found statistically significant improvements for overall healthy choices $t$ $(287)=2.98, p=0.003$ [10].

Katz et al. [10] implemented a nutrition education program with the purpose of educating elementary students to differentiate between more healthful and less healthful food choices in a variety of categories. A total of 1,180 second, third and fourth grade students from three separate schools participated and randomly assigned to groups. A control group of 552 students and an intervention group of 628 students were identified. The program was conducted by the school's physical education teachers over several sessions. Education included reading food labels and identification the more healthy food choice. Parents were also included in the nutrition education, as information was sent home and included at all school functions.

The intervention, titled The Nutrition Detectives, focused on outcomes of nutrition and two other areas, dietary patterns and BMI. The program utilized a food literacy questionnaire to assist with evaluation of the nutrition education given. The 10 questions focused on students making a more healthful choice. The students used the nutrition information learned from the program to choose the food label that would be considered the more healthy choice [11].

Data collected using a pre/post-test method showed a statistically significant improvement of nutritional knowledge from baseline at $18.1 \%$ to completion at $26.9 \%$ respectively, $\mathrm{p}<0.01$. Grade 3 students showed the most nutritional knowledge improvement. Parents of students also showed a statistically significant improvement. Dietary patterns of students were not statistically significant, when looking at food choices in specific categories. Overall, this study provided fundamental evidence that the nutrition detectives program could be effective to increase students' knowledge in making more healthful choices [11].

\section{Project Methods}

\section{Description}

The project used the Nutrition Detectives program to educate teachers about the importance of healthy lifestyle choices and habits. The program was developed by David Katz, MD and his wife Catherine Katz, PhD, for elementary school children. It is a resource to educate and engage children in a minimal amount of time. This program was developed to educate children on how to read food labels and how to use this information to make healthful food choices. 


\section{Measures and instruments}

The Food Label Literacy for Applied Nutritional Knowledge (FLLANK) was used to assess the teacher's knowledge of healthy food choices. The FLLANK is a 10 item paper and pen test constructed to measure the nutrition knowledge of elementary school children. It is based on food label subject matter found in the Nutrition Detectives program [12]. Pairs of food labels are presented and participants select the healthier choice or "I can't tell." The instrument has previously established reliability, Cronbach's alpha of 0.77 for use with children mean age 8.6 years. Intra-class correlation coefficient (ICC, 0.68) was determined by comparison with the Overall Nutritional Quality Index ONQI, [12]. Results showed the FLLANK questionnaire was both a reliable and valid measurement instrument for the Nutrition Detectives food label literacy program.

\section{Implementation}

All second grade teachers and health/PE teachers at Murray Elementary were offered the opportunity to participate. A verbal script was used to recruit participants. Prior to implementation, all participants were given a cover letter and completed a demographic survey. The purpose of the program and expectations of the participants was discussed and participants were asked to complete the FLLANK pre-test.

Once all pre-implementation information was collected, lesson one of the program was completed. Lesson one included information about "If you are what you eat, then what in the world are you?" [13] this included information about the importance of food choices and health. Discussion about what makes food good for you and risk factors pertaining to chronic disease, fitness and weight control were also included. This is the initial lesson and focused on activities to promote interaction of participants. Participants were reminded about the meeting next week when lesson two, three and four would be discussed.

Week two consisted of lesson two, three and four. Lesson two was "Polar bears in the Sahara." The discussion included things discussed in lesson one, and compared those to the struggle of polar bears in the heat of the Sahara desert. This lesson focused on sugar, salt and fat and helped to increase knowledge of the importance of overcoming the obstacles to ensure healthy eating. Lesson three included Supermarket Spy Kids. This section is described by [12] as the "heart of the program". This lesson included information to help participants to interpret food labels. Four areas were emphasized: (1) partially hydrogenated oil; (2) high fructose corn syrup; (3) fiber; and (4) the length of the ingredient list. These four areas provided direction for making better overall nutritional choices. Lesson four gave an opportunity for hands on experience with food choices. "Let's play with our food" is the portion of the program where teachers were divided into eight groups and one shopping bag of items was given to group. Clues from information previously presented were used to identify more healthful choices. At the end of the lesson participants were allowed to taste the ingredients and see that there are good tasting options for healthful choices.
Week three included lesson five and a review of the Nutrition Detective's clues. Lesson five was food for thought: take away messages. It was used to point out that some foods, such as fresh produce do not have labels, but are always a good choice. It also provided a summary of the key points. A question and answer forum was conducted and the FLLANK post-test was given after discussions concluded.

\section{Results}

\section{Sample/participants}

Data were analyzed using Statistical Package for Social Sciences (SPSS) Version 21. A convenience sample $(\mathrm{N}=9)$ of educators was obtained. The 9 participant's age ranged from 26 to 63 years with a mean age of 45.33 (Table 1). Years of education experience ranged from 3 to 22 years with a mean of 14.67 (Table 1).

Table 1: Participant demographics.

\begin{tabular}{|l|c|c|c|}
\hline Variable & N & Range & Mean + SD \\
\hline Age & 9 & 26 to 63 & $45.33+10.30$ \\
\hline Years of Experience & 9 & 3 to 22 & $14.67+5.32$ \\
\hline
\end{tabular}

\section{Food label literacy for applied nutritional knowledge (FLLANK)}

A paired-samples t-test was conducted to evaluate for mean difference in the FLLANK pre and post-test. Results showed a statistically significant increase in overall scores from preeducation ( $M=6.44 \pm 1.67$ ) to post-education ( $M=9.11 \pm 0.782)$, $t$ $(8)=-8.0, p<.001$ (two-tailed). The mean increase in FLLANK scores was -2.67 with a $95 \% \mathrm{Cl}$ ranging from -3.435 to -1.898 . The magnitude of effect was large (eta squared $=0.70$ ) (Table 2 ).

Table 2: Paired t-test Comparison of Mean Scores of the Food Label Literacy for Applied Nutritional Knowledge (FLLANK).

\begin{tabular}{|l|l|c|c|c|}
\hline Score & Mean + SD & t & df & p \\
\hline FLLANK pre-test $(n=9)$ & $6.44+1.67$ & -8 & 8 & $<0.001$ \\
\hline FLLANK post-test $(n=9)$ & $9.11+0.782$ & & & \\
\hline
\end{tabular}

\section{Conclusion}

In conclusion this project promoted the use of education to elementary school teachers. This project utilized a variety of different protocols and educational tools to ensure that participants have the knowledge to make healthy lifestyle choice, including healthy food choices. This project can be continued each year in the school system, therefore making it sustainable over time. The potential impact of this project included the specific participants and the community in which they live. This project will help to improve the community fight against obesity that is currently taking place in the Murray Kentucky area. 


\section{References}

1. Leventer-Roberts M, Patel A, Trasande L (2012) Is severity of obesity associated with diagnosis or health education practices? Int J Obes, 36: 1571-1577.

2. Freedman D, Wang J, Thornton J (2009) Classification of body fatness by body mass index for age categories among children. Arch Pediatr Adolesc Med, 163: 801-811.

3. Wijg AH, Scholtens S, Bemelmans WJE, de Jongste JC, Kerkhof JC, et al. (2010) Comorbidities of obesity in school children: A cross sectional study in the PIAMA birth cohort. BMC Public Health, 10: 184-193.

4. Mahmood L (2015) The childhood obesity epidemic: A mini review. Int J Med 5: 6-9.

5. Savinon C, Taylor J S, Canty-Mitchell J, Blood-Siegfried, J (2012) Childhood obesity: Can electronic medical records customized with clinical practice guidelines improve screening and diagnosis? J Am Acad Nurse Pract, 24: 463-471.

6. Krisberg K (2006) Food marketing toward youth contributing to unhealthy choices. Nation's Health, 36: 19-29.
7. Falter RA, Pignotti-Dumas K, Popish SJ, Petrelli HMW, et al. (2011) A service learning program in providing nutrition education to children. Am J Pharm Educ, 75: 1-7.

8. Ling J, King KM, Speck BJ, Kim S, Wu D (2014) Preliminary assessment of a school-based healthy lifestyle intervention among rural elementary school children. J Schl Health, 84: 247-255.

9. Prosper MH, Moczulski VL, Qureshi A, Weiss M, Bryars T (2009) Healthy for life/PR4ME: Assessing an intervention targeting childhood obesity. Calif J Health Promot, 7: 23-32.

10. Katz DL, Katz CS, Treu JA, Reynolds J, Kjike V, et al. (2011) Teaching healthful food choices to elementary schools students and their parents; the nutrition detectives program. J Sch Heal 81: 21-28.

11. Reynolds JS, Treu JA, Valentine N, Walker J, Smith E, et al. (2012) The Validation of a food label literacy questionnaire for elementary school children. J Nutr Educ Behav 44: 262-266.

12. Katz DL, Katz C (2009). Nutrition detectives teacher's manual. Derby, CT: Yale-Griffin Prevention Research Center. 